\title{
Comparison of Precision and Accuracy of Five Methods to Analyse Total Score Data
}

\author{
Gustaf J. Wellhagen, ${ }^{1}$ Mats O. Karlsson, ${ }^{1}$ and Maria C. Kjellsson ${ }^{1,2}$ (1)
}

Received 17 September 2020; accepted 1 December 2020; published online 17 December 2020

\begin{abstract}
Total score (TS) data is generated from composite scales consisting of several questions/items, such as the Movement Disorder Society-Unified Parkinson's Disease Rating Scale (MDS-UPDRS). The analysis method that most fully uses the information gathered is item response theory (IRT) models, but these are complex and require item-level data which may not be available. Therefore, the TS is commonly analysed with standard continuous variable $(\mathrm{CV})$ models, which do not respect the bounded nature of data. Bounded integer (BI) models do respect the data nature but are not as extensively researched. Mixed models for repeated measures (MMRM) are an alternative that requires few assumptions and handles dropout without bias. If an IRT model exists, the expected mean and standard deviation of TS can be computed through IRT-informed functions-which allows CV and BI models to estimate parameters on the IRT scale. The fit, performance on external data and parameter precision (when applicable) of CV, BI and MMRM to analyse simulated TS data from the MDS-UPDRS motor subscale are investigated in this work. All models provided accurate predictions and residuals without trends, but the fit of $\mathrm{CV}$ and BI models was improved by IRT-informed functions. The IRT-informed BI model had more precise parameter estimates than the IRT-informed CV model. The IRT-informed models also had the best performance on external data, while the MMRM model was worst. In conclusion, (1) IRT-informed functions improve TS analyses and (2) IRT-informed BI models had more precise IRT parameter estimates than IRT-informed CV models.
\end{abstract}

KEY WORDS: composite scale data; total score data; bounded integer model; mixed models for repeated measures; IRT-informed total score analysis.

\section{BACKGROUND}

Composite scale data is made up of many questions/ items with categorical responses that can be summed up through an algorithm into a total score (TS), which is discrete and bounded. Parkinson's disease is a therapeutic area where no reliable biomarker exists to monitor disease progression and treatment efficacy. Instead, composite scales designed for diagnosis are used for these purposes.

Item response theory (IRT) models make use of itemlevel information; therefore, well-constructed IRT models are considered the most informative way of analysing such data. They map the disease severity to one or several latent variable $(\mathrm{s})(\Psi)$. However, they are complex to develop, may be difficult to estimate due to a large number of parameters,

\footnotetext{
${ }^{1}$ Pharmacometrics Research Group, Department of Pharmacy, Uppsala University, Box 580, 751 23, Uppsala, Sweden.

${ }^{2}$ To whom correspondence should be addressed. (e-mail: maria.kjellsson@farmaci.uu.se)
}

may take long time to run and sometimes the item-level data is unavailable. The modeller can then turn to alternative models: continuous variable (CV), bounded integer (BI) (1) or less mechanistic models such as mixed models for repeated measures (MMRM).

Different models have different strengths and weaknesses; CV models are commonplace and easy to implement, but they do not respect the scale boundaries nor the discrete nature of the TS data; BI models respect the boundaries and data nature by operating on a latent variable scale $(Z)$ but have not been used as extensively; MMRM is an appropriate alternative to analysis of (co)variance (ANOVA/ANCOVA) in the case of missing data (2-4), where few assumptions need to be made about the response, but requires many parameters to be estimated and the models do not lend themselves to extrapolation. Beta regression (5), an alternative to standard Gaussian CV models, is sometimes used for bounded outcomes (6), which allows for flexible distributions (J- or U-shaped) (7-12). However, this requires a transformation of the data, and besides, the transformation is onto the open interval $(0,1)$ so that the boundaries are not included, which is typically achieved through $Y^{*}=(Y \cdot(n-1)+0.5) / n$, where $n$ is 
the sample size (5). The choice of transformation or correction factor has a large impact on the data at or close to the boundaries, and beta regression has been shown to be statistically non-rigorous (13) and behaves poorly at the boundaries (14). Logit transformation (15) is another option to constrain an outcome to $(0,1)$, which also faces issues at the boundary since these are only asymptotes.

If an IRT model exists for the scale in question, IRTinformed functions of disease progression and standard deviation (SD) can be computed through the item characteristic curves. With these link functions, the goodness-of-fit of $\mathrm{CV}$ and BI models for TS analyses can be improved, the latent variable parameters for disease severity of an IRT model can be captured and the relative information of different model types can be compared (Wellhagen GJ, Ueckert S, Kjellsson MC, Karlsson MO. An item response theory-informed strategy to model total score data from composite scales. Forthcoming 2020).

Comparisons of MMRM and CV models have been performed on TS data within the field of Alzheimer's disease $(16,17)$. It was found that MMRM models are often overparameterised but provide tighter confidence intervals around treatment effects. Since treatment effects are often the primary interest in the late-stage drug development, this is an appealing option to get more certain predictions of drug effect sizes.

In this work, we illustrate the strengths and weaknesses of CV, BI and MMRM models to analyse TS data in a phase 3 clinical trial setting in Parkinson's disease via simulations from an IRT model. The IRT-informed functions are also evaluated in $\mathrm{CV}$ and $\mathrm{BI}$ models to improve fit, increase precision and reduce bias of IRT parameters. Also, the precision and bias in the drug effect at end-of-treatment is investigated.

\section{METHODS}

\section{Simulation Model}

A previously published IRT model (18) was used to simulate MDS-UPDRS motor data during 42 months, across 10 visits. A Weibull dropout model was added, see Eqs. (1-2):

$$
\left\{\begin{array}{c}
\rho=\theta_{1} e^{-\theta_{2} \frac{t}{12}} \\
P(t)=1-e^{-\left(\ln (2) \frac{t}{\rho}\right)^{\theta_{3}}}
\end{array}\right.
$$

where $\rho$ is the scale factor, $\theta_{1}$ is the baseline mean time to dropout (120 months), $\theta_{2}$ is the hazard ratio for the time in the study $\left(0.03\right.$, i.e. $\sim 3 \%$ per year), $\theta_{3}$ is the shape factor (set to 2), $t$ is time in months and $P(t)$ is then the resulting probability of dropping out at time $t$. The baseline hazard was also associated with an inter-individual variability (IIV) (proportional with variance: $\omega^{2}=0.25$ ). The resulting dropout rate $(\sim 15 \%)$ was similar to previously reported in studies of at least 10 weeks (19).

The disease progression was assumed to be linear on the latent variable scale, as was reported in the published IRT model (18).

\section{Simulation Scenarios}

Four different populations were simulated:

1. Relatively healthy $\left(\Psi_{\text {baseline }}=0\right)$ with slow disease progression (slope $=0.3 /$ year)

2. Relatively healthy with fast disease progression (slope $=0.6 /$ year $)$

3. Relatively ill $\left(\Psi_{\text {baseline }}=1.5\right)$ with slow disease progression (slope $=0.3 /$ year)

4. Relatively ill with fast disease progression ( year)

For each population, three different kinds of drug effects were implemented $v s$. placebo:

a. A disease-modifying effect of $30 \%$ reduction of the slope

1 A symptomatic effect, e.g. offset, on the latent variable with a reduction of 0.315 and 0.63 , for patients with slow and fast progression, respectively

2 A combination of (a) and (b) with $15 \%$ slope reduction and a reduction of 0.1575 and 0.315 , for patients with slow and fast progression, respectively

The baseline and slope had additive IIVs with $\omega^{2}=0.5$ and $\omega^{2}=0.025$ respectively, while the drug effects all had proportional IIVs with $\omega^{2}=0.05$. All drug effects were titrated such that they would result in the same absolute difference to placebo at month 42 , independent of the drug effect being disease-modifying or symptomatic and patients having a high or low baseline: a difference of 0.315 or 0.63 for patients with slow or fast disease progression, respectively. Drug effects came into act immediately post-baseline. For each scenario $(4 \times 3=12), 100$ simulations were run, totalling 1200 studies.

A validation data set containing 1000 individuals (1:1 design) at 10 occasions each (with dropout) was also simulated for each simulation scenario.

\section{Titration of Study Size}

The power was titrated to be the same in all studies. In each of the 12 simulation scenarios, the number of individuals needed per treatment group to identify a drug effect with $80 \%$ power at a $5 \%$ significance level at 42 months was calculated and rounded up to the nearest 5. A 1:1 parallel design placebo-controlled trial with $n$ /arm set to the titrated value was then analysed for each simulation number.

\section{Estimation Models}

The simulated data were analysed once with each of five different models: (1) standard CV (S-CV), (2) IRT-informed CV (I-CV), (3) standard BI (S-BI), (4) IRT-informed BI (I$\mathrm{BI}$ ) and (5) MMRM with 1st-order autoregressive residual correlation model (AR1). The definitions for all nonlinear mixed-effects (NLME) models are the same as in (Wellhagen GJ, Ueckert S, Kjellsson MC, Karlsson MO. An item response theory-informed strategy to model total score data from composite scales. Forthcoming 2020), while the MMRM model is only described here. 


\section{Continuous Variable Models}

In the standard $\mathrm{CV}$ model $(\mathrm{S}-\mathrm{CV})$, the observation $j$ for subject $i$ at time $t_{i j}$ is described through:

$$
\begin{aligned}
Y_{i j}= & f\left(\theta, \eta_{i}, t_{i j}, X_{i}\right)+\varepsilon_{i j} \\
& \eta_{i} \sim N\left(0, \omega^{2}\right) \\
& \varepsilon_{i j} \sim N\left(0, \sigma^{2}\right)
\end{aligned}
$$

where $\theta$ is the fixed effect parameters, $\eta_{i}$ is the random effects of the inter-individual, $X_{i}$ is the covariates, $\varepsilon_{i j}$ is the residual unexplained variability (RUV), $\omega^{2}$ is the variance of the IIV and $\sigma^{2}$ the variance of the RUV.

The fully IRT-informed CV model (I-CV) is expressed as:

$$
\begin{gathered}
\Psi_{i j}=h\left(\theta, \eta_{i}, t_{i j}, X_{i}\right) \\
Y_{i j}=p n_{1}\left(\Psi_{i j}\right)+\varepsilon_{i j} \cdot p n_{2}\left(\Psi_{i j}\right) \\
\eta_{i} \sim N\left(0, \omega^{2}\right) \\
\varepsilon_{i j} \sim N(0,1)
\end{gathered}
$$

where $\Psi_{i j}$ is a latent variable described by the nonlinear function $h(\cdot)$ and $p n_{1}$ as well as $p n_{2}$ are predetermined polynomials (Wellhagen GJ, Ueckert S, Kjellsson MC, Karlsson MO. An item response theory-informed strategy to model total score data from composite scales. Forthcoming 2020). The other variables maintain their definition from above.

\section{Bounded Integer Models}

The standard BI model (S-BI) is a discrete data model, where the probability of an individual $i$ to have the score $k$ at time $t_{i j}$ is:

$$
P\left(Y_{i j}=k\right)=\phi\left(\frac{Z_{\frac{k}{n}}-f\left(\theta, \eta_{i}, t_{i j}, X_{i}\right)}{g\left(\sigma, \eta_{i}, t_{i j}, X_{i}\right)}\right)-\phi\left(\frac{Z_{\frac{k-1}{n}}-f\left(\theta, \eta_{i}, t_{i j}, X_{i}\right)}{g\left(\sigma, \eta_{i}, t_{i j}, X_{i}\right)}\right)
$$

where $\phi$ is the cumulative distribution function for the standard normal distribution, $Z_{\mathrm{k} / \mathrm{n}}$ and $Z_{(\mathrm{k}-1) / \mathrm{n}}$ are the cut points between categories $k$ and $k-1$ defined through the probit function for an $n$-category scale, $f(\cdot)$ is the function for the mean and $g(\cdot)$ the function for the variance on the probit scale. For all BI models, the special cases for the first and last categories $(k=1, k=n)$ apply:

$$
\begin{aligned}
& P\left(Y_{i j}=1\right)=\phi\left(\frac{Z_{\frac{1}{n}}-f\left(\theta, \eta_{i}, t_{i j}, X_{i}\right)}{g\left(\sigma, \eta_{i}, t_{i j}, X_{i}\right)}\right) \\
& P\left(Y_{i j}=n\right)=1-\phi\left(\frac{Z_{\frac{n-1}{n}}-f\left(\theta, \eta_{i}, t_{i j}, X_{i}\right)}{g\left(\sigma, \eta_{i}, t_{i j}, X_{i}\right)}\right)
\end{aligned}
$$

The fully IRT-informed BI model (I-BI) is expressed as:

$$
\begin{gathered}
\Psi_{i j}=h\left(\Theta, \eta_{i}, t_{i j}, X_{i}\right) \\
P\left(Y_{i j}=k\right)=\phi\left(\frac{Z_{\frac{k}{n}}-p n_{3}\left(\Psi_{i j}\right)}{p n_{4}\left(\Psi_{i j}\right)}\right)-\phi\left(\frac{Z_{\frac{k-1}{n}}-p n_{3}\left(\Psi_{i j}\right)}{p n_{4}\left(\Psi_{i j}\right)}\right) \\
\eta_{i} \sim N\left(0, \omega^{2}\right)
\end{gathered}
$$

where $p n_{3}$ as well as $p n_{4}$ are predetermined polynomials (distinct from $p n_{1}$ and $p n_{2}$ ).

\section{Mixed Models for Repeated Measures}

The MMRM model is defined as:

$$
\begin{gathered}
Y_{i j m}=\theta_{j m}+\eta_{i}+\varepsilon_{i j} \\
\eta_{i} \sim N\left(0, \omega^{2}\right) \\
\varepsilon_{i j} \sim N\left(0, \sigma_{j}^{2}\right)
\end{gathered}
$$

where $Y_{i j m}$ is the response of individual $i$ at time $j$ and dose arm $m, \theta_{j m}$ the fixed effect, $\eta_{i}$ the random effect of the interindividual variability (IIV), $\omega^{2}$ the variance of the IIV, $\varepsilon_{i j}$ the residual unexplained variability (RUV) and $\sigma_{j}^{2}$ the variance of the RUV. A 1st-order residual correlation was assumed. Two alternative models were tested: either the variance was allowed to vary between the dose arms $\left(\sigma_{m}{ }^{2}, 2\right.$ parameters) or between each visit $\left(\sigma_{j}^{2}, 10\right.$ parameters).

\section{Evaluation Metrics}

Precision and accuracy of estimated parameters were investigated for the two IRT-informed models (I-CV and I$\mathrm{BI})$, where the parameters were expressed on the same scale as the IRT model, i.e. on the latent variable $\Psi$. The precision and accuracy were illustrated by the distribution of the parameter estimates.

Model fit was evaluated through Akaike information criterion (AIC), computed from Objective Function Value (OFV) as in Eq. (3):

$$
\mathrm{AIC}=\mathrm{OFV}+2 p
$$

where $p$ is the number of parameters (including IIVs) estimated in the model. The largest model was the MMRM with variance per time point, while the smallest were the I$\mathrm{CV}$ and I-BI since no SD was estimated then. As an example, given a symptomatic drug effect, the S-CV and S-BI models had a total of 8 parameters, the I-CV and I-BI had 6, while the MMRM models had 26 and 34, respectively. The AIC for the S-CV model was used as a reference to compute relative AIC for all other models. The performance on external data of all estimation models, with the final parameter estimates fixed, was also evaluated in the validation data set-via relative OFV since no parameters were estimated.

The goodness-of-fit was also assessed by assessing the proportion of observations, with a residual outside \pm 2 SD. 
Under a standardised residual following $N(0,1)$, this number should be $5 \%$. Different measurements of residuals were used for different models due to the nature of the models. For the $\mathrm{CV}$ and MMRM models (continuous models), the conditional weighted residual (CWRES) (20) was used and for the BI models (likelihood models), the Pearson individual weighted residual (PIWRES) was used (21). PIWRES should be a standardised residual $(N(0,1))$.

Lastly, the precision and bias of the predicted drug effect at the end-of-study were evaluated with the final parameter estimates; the placebo-corrected prediction of TS ( $\Delta$ TS) was simulated for each scenario with 500 individuals in a crossover design.

\section{Software}

The CV and BI models were evaluated through nonlinear mixed-effects modelling with NONMEM version 7.4 (ICON Development Solutions, Ellicott City, MD), executed through PsN version 4.9 (22,23). The Laplacian estimation method with $\eta-\varepsilon$ interaction was used for all the $\mathrm{CV}$ and MMRM models, while BI models were estimated with stochastic approximation and expectation maximisation (SAEM). Importance sampling with an expectation step was added after the estimation step to generate comparable OFVs for all models. Graphics were made with R version 3.6.2 (24) and tidyverse (25). The piraid (26) package was used to create the IRT-informed functions, including the predetermined polynomials $\left(\mathrm{pn}_{1}-\mathrm{pn}_{4}\right)$ and associated NONMEM control streams.

\section{RESULTS}

The sample size, calibrated to ca $80 \%$ power, varied from 25 to 80 subjects/arm depending on the simulation scenario. The lowest sample size was associated with a relatively healthy population with fast disease progression and the highest sample size was needed for the relatively ill population with slow disease progression. In Supplemental Table SI, sample sizes for all the scenarios are tabulated.

For the two MMRM models assessed, a different variance per arm or per time point, the average AIC was always lower for the larger, with variance per time point, model (results not shown). The MMRM models were also evaluated in $\mathrm{R}$ (results not shown), where an additional model, with unconstrained residual correlation matrix, was tested. This model did however not improve the fit compared to the AR1 model. Hence, the MMRM model with AR1 and variance per time was chosen for further comparison.

In Fig. 1, the averages of the observed and predicted total score for all models are shown for one simulation to exemplify: a symptomatic drug effect. There was no sign of bias and the precision was similar for all models. Similar plots with examples for the other drug effects are shown in Supplemental Fig. S1 and Supplemental Fig. S2.

In Fig. 2 and Supplemental Fig. S3, the distributions of the baseline and slope parameters in the IRT-informed models are shown. Neither of the models showed a strong bias in these parameter estimates; however, the I-BI model has a few cases of overprediction of the slope-when simultaneously showing overpredicted disease-modifying drug effect parameter (Supplemental Fig. S6). The I-BI model had more precise estimates of the baseline parameters than the I-CV model. The relative bias of the IIV for these parameters was comparable, shown in Supplemental Fig. S4 and Supplemental Fig. S5.

In Fig. 3 and Supplemental Fig. 6, the distributions of the symptomatic and disease-modifying drug effect parameters in the IRT-informed models are shown. The precision of the estimates from the IRT-informed models was similar, but the I-CV model tended to underpredict the symptomatic drug effect parameter when the disease progression was slow, with a more pronounced bias in the combined drug effect. As seen in Supplemental Fig. S7 and Supplemental Fig. S8, both models showed signs of positive bias in estimating the variance of IIV; however, the I-BI model gave considerably more precise estimates. The underprediction may also be a result of these IIVs being implemented lognormally, which means that the median is lower than the mean.

In Fig. 4, the residual diagnostic for all models across all visits is shown for one example: a symptomatic drug effect. It can be seen that the BI models typically show less than $5 \%$ residuals outside 2 standard deviations, while the $\mathrm{CV}$ and MMRM models target this number. There is no strong temporal trend, but there are more outliers at the end of the study. Similar plots for the other drug effects are shown in Supplemental Fig. S9 and Supplemental Fig. S10.

In Fig. 5, the AIC of all models relative to the $\mathrm{S}-\mathrm{CV}$ model is shown. The IRT-informed models (I-CV and I-BI) were superior to the standard models and on par with each other. The S-BI offered a better fit than the S-CV model as judged by the relative AIC. The MMRM model had the poorest fit. In Fig. 6, the relative OFV of all models (again compared to the S-CV model) to external data with the final estimates is shown. The same trends were visible there: IRTinformed models had the best fit and MMRM the worst.

In Fig. 7, the predicted and true difference from placebo at end-of-study is shown. Mostly, the predictions were close to the true values. The MMRM model always had unbiased predictions, but they were the most imprecise of all, while the $\mathrm{S}-\mathrm{CV}$ had the most biased predictions. The I-CV model, however, showed no signs of bias. The S-BI and I-BI had a few cases of small bias. It should be noted that the BI models are the only models that predict real life-like data: as integer values.

\section{DISCUSSION}

All the investigated models provided similar predictions, but the possibility to use IRT-informed functions provided improvements in both goodness-of-fit (AIC), due to a better description of the disease progression and SD, as well as performance on external data. In addition, as the IRTinformed functions transform the estimates of the model to the IRT-disease progression scale, parameter estimates can be compared between different model types. The I-CV model was better than the I-BI model at predicting the size of the drug effect without bias, while their respective precision was similar.

For a modeller dealing with TS data, the purpose of the analysis may lead to different model choices. If the goal is to determine a statistical difference between two treatments at 


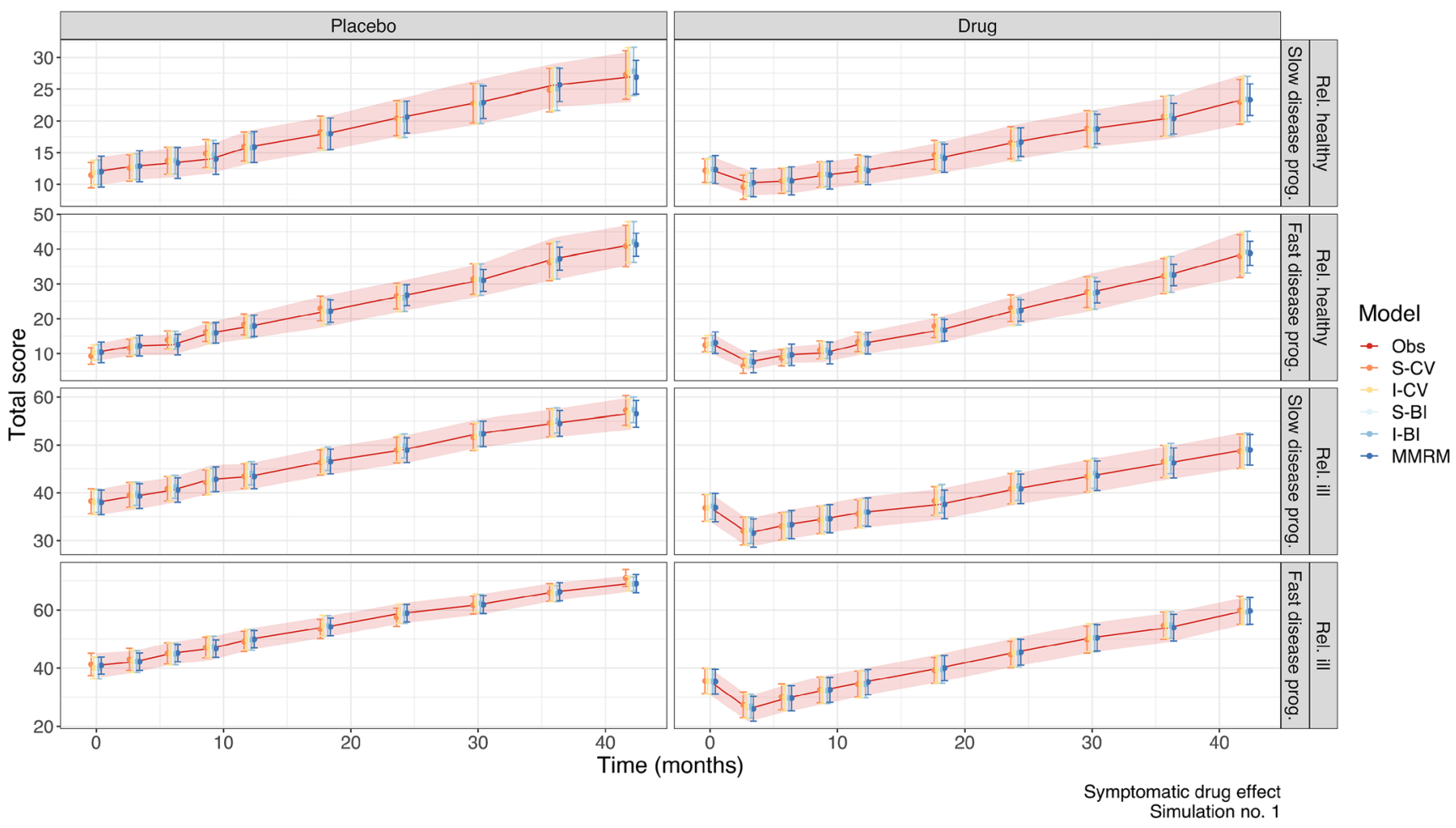

Fig. 1. Average and $95 \%$ prediction interval (PI) of observations and predictions at each time point for all models, following a symptomatic drug effect for simulation number 1 of 100, stratified by population. The solid line represents the average of the observations and the shaded area represents the PI of the observations. Points represent the average of the predictions for each model and error bars represent the PI of the predictions for each model, with different colours. IRT, item response theory; I-BI, IRT-informed bounded integer model; I-CV, IRT-informed continuous variable model; S-BI, standard bounded integer model; S-CV, standard continuous variable model; MMRM, mixed model for repeated measures

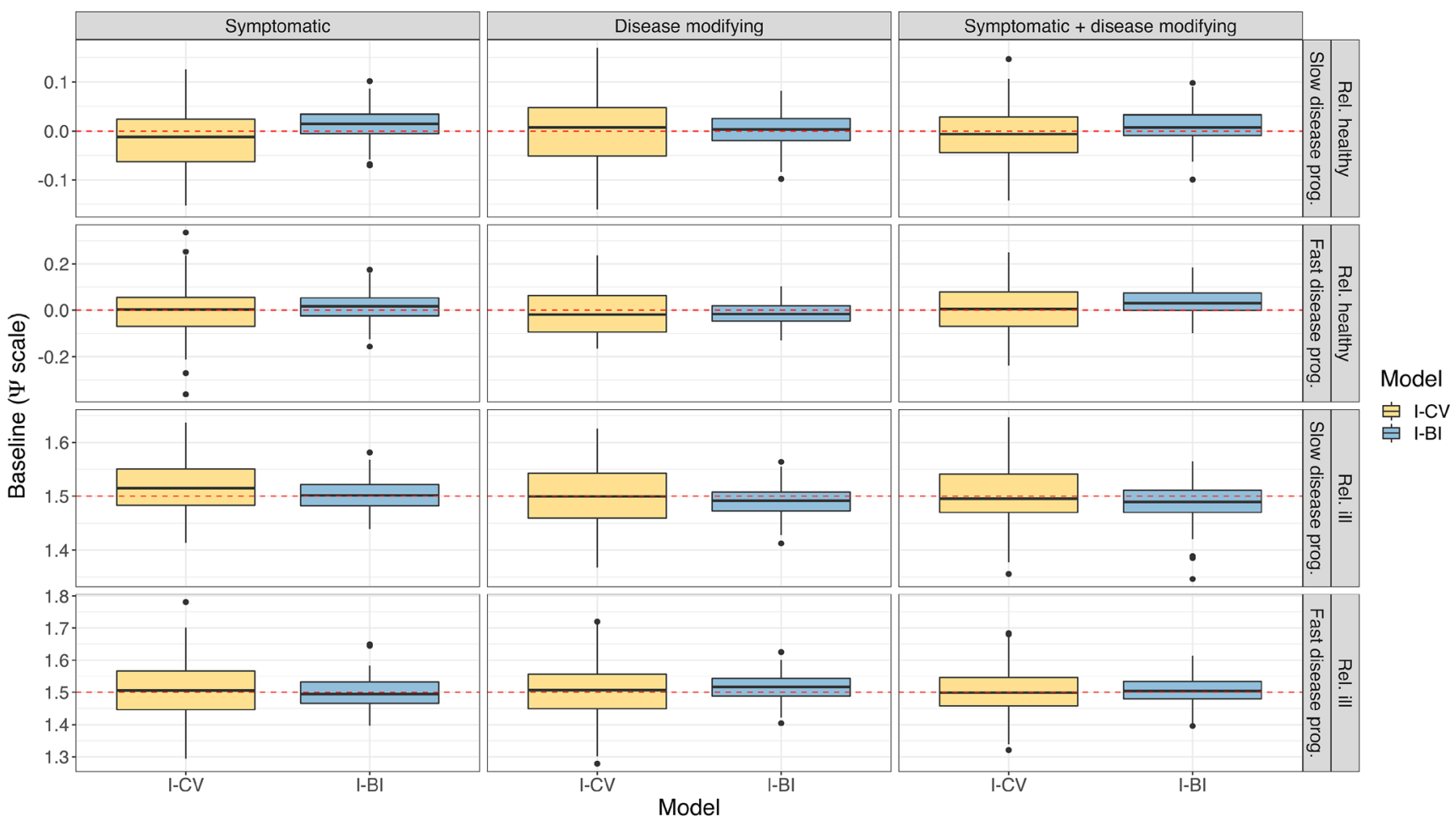

Fig. 2. Distribution of the baseline parameter in IRT-informed models, stratified by drug effect and population. The dashed red line indicates the true parameter value. IRT, item response theory; I-CV, IRT-informed continuous variable model; I-BI, IRT-informed bounded integer model 


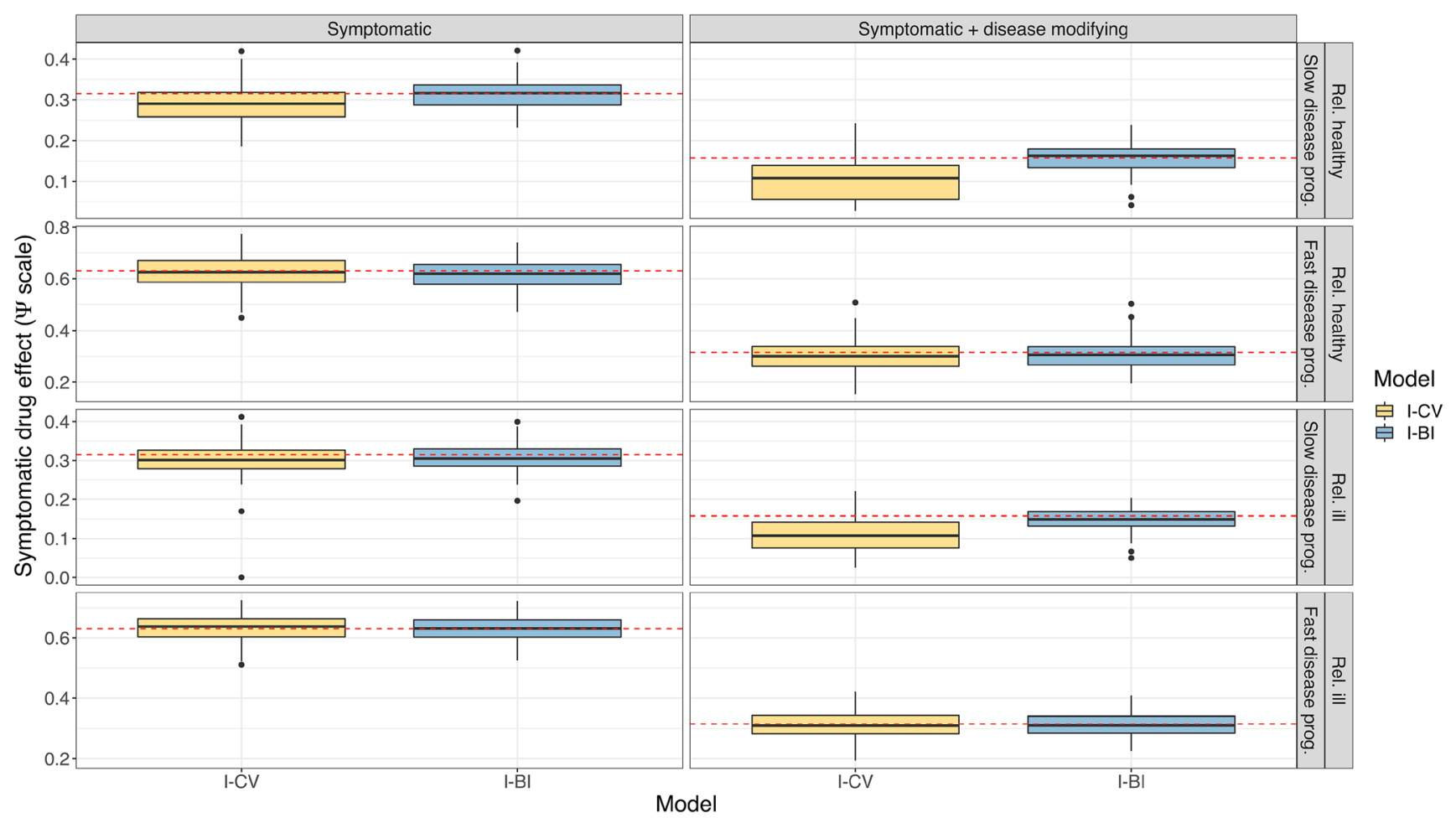

Fig. 3. Distribution of the symptomatic drug effect parameter in IRT-informed models, stratified by drug effect and population. The dashed red line indicates the true parameter value. IRT, item response theory; I-CV, IRT-informed continuous variable model; I-BI, IRT-informed bounded integer model

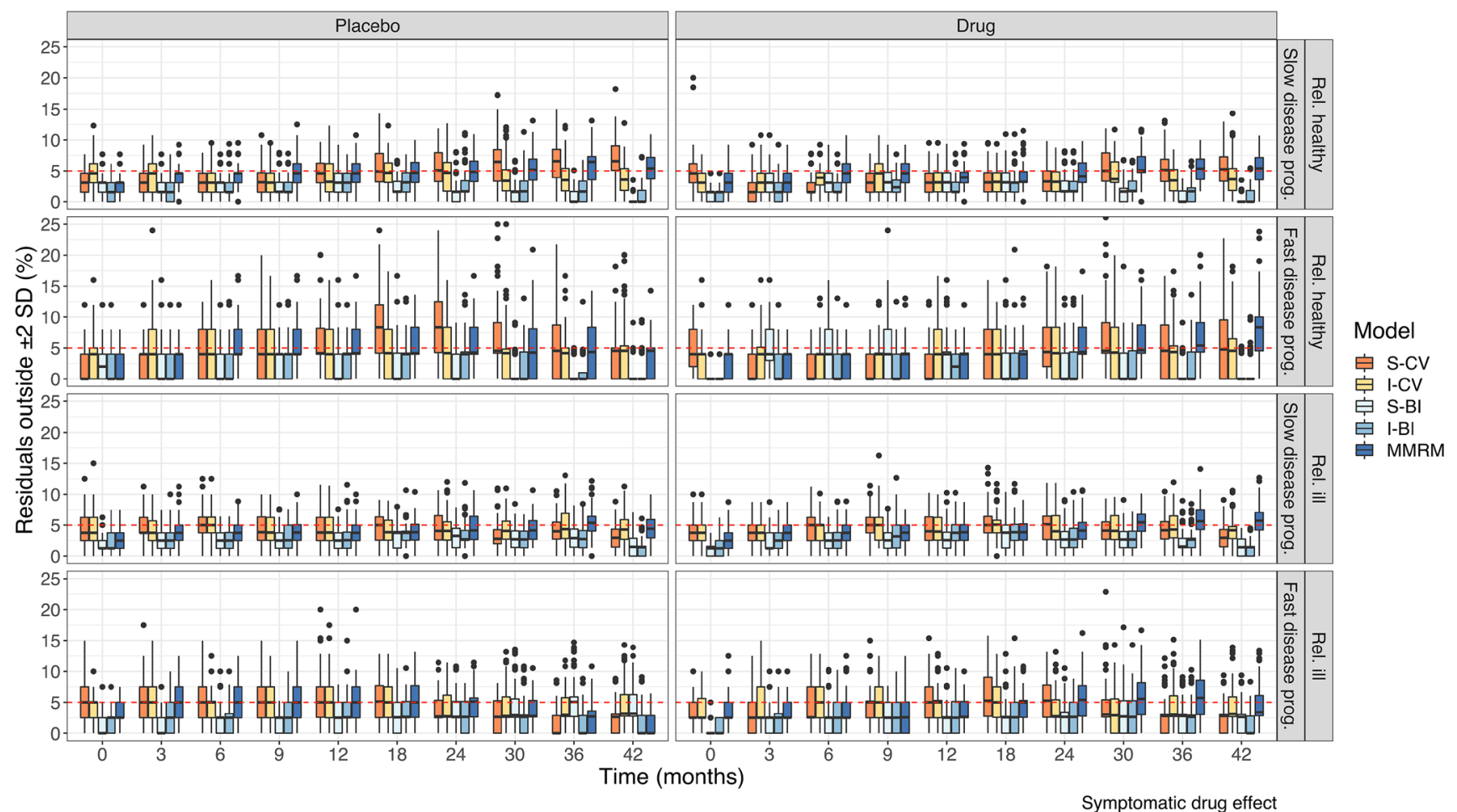

Fig. 4. Residual diagnostic showing the percent residuals outside \pm 2 standard deviations for all models under a symptomatic drug effect, stratified by population. Note that the y-axis has been cut for visibility. CWRES, conditional weighted residual; IRT, item response theory; I-BI, IRT-informed bounded integer model; I-CV, IRT-informed continuous variable model; MMRM, mixed model for repeated measures; PIWRES, Pearson individual weighted residual; S-BI, standard bounded integer model; S-CV, standard continuous variable model 


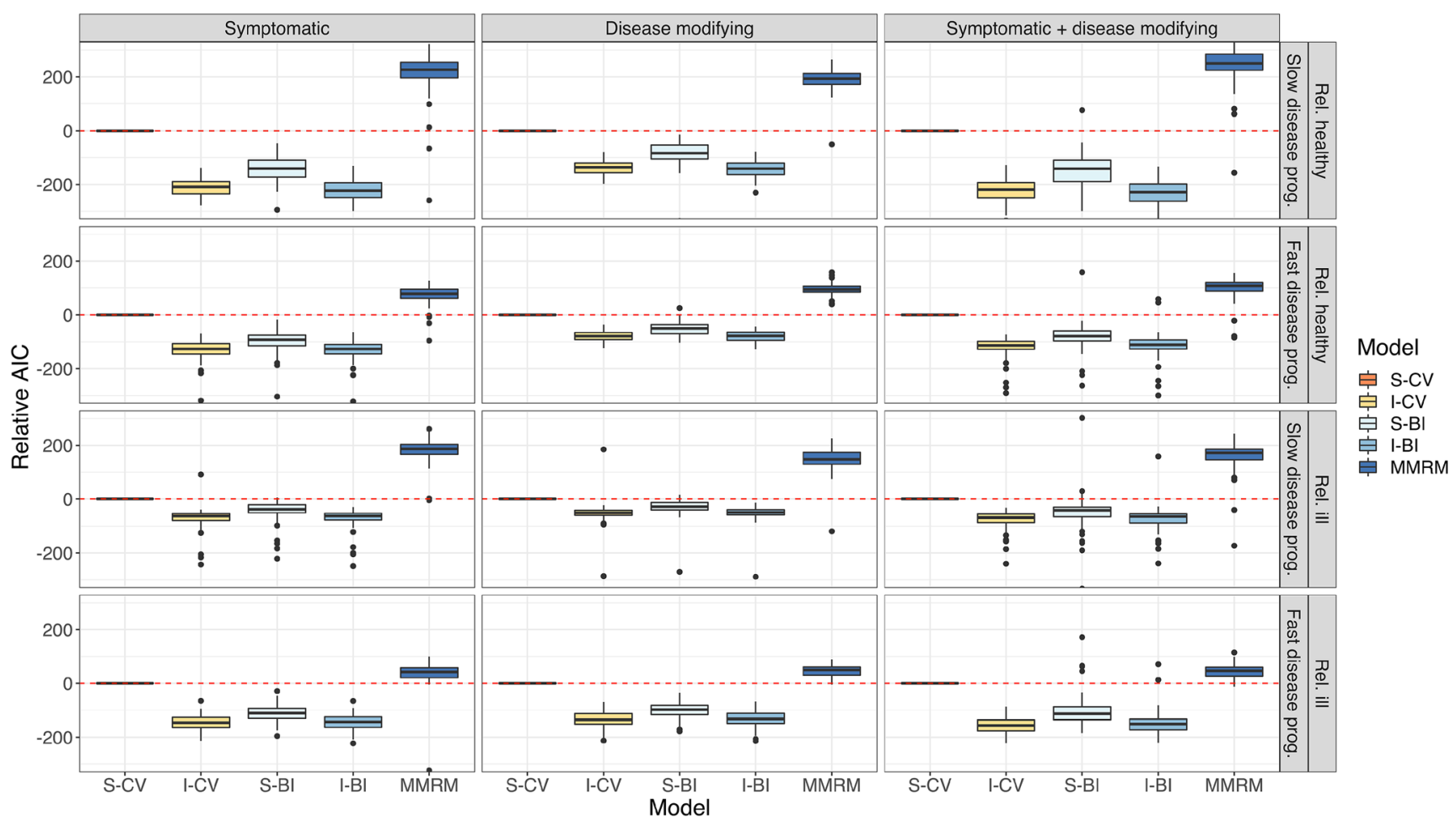

Fig. 5. Relative AIC of the investigated models, stratified by drug effect and population. The standard continuous variable (S-CV) model is used as the reference model. Note that the y-axis has been cut for visibility. IRT, item response theory; I-BI, IRT-informed bounded integer model; I-CV, IRT-informed continuous variable model; S-BI, standard bounded integer model; MMRM, mixed model for repeated measures

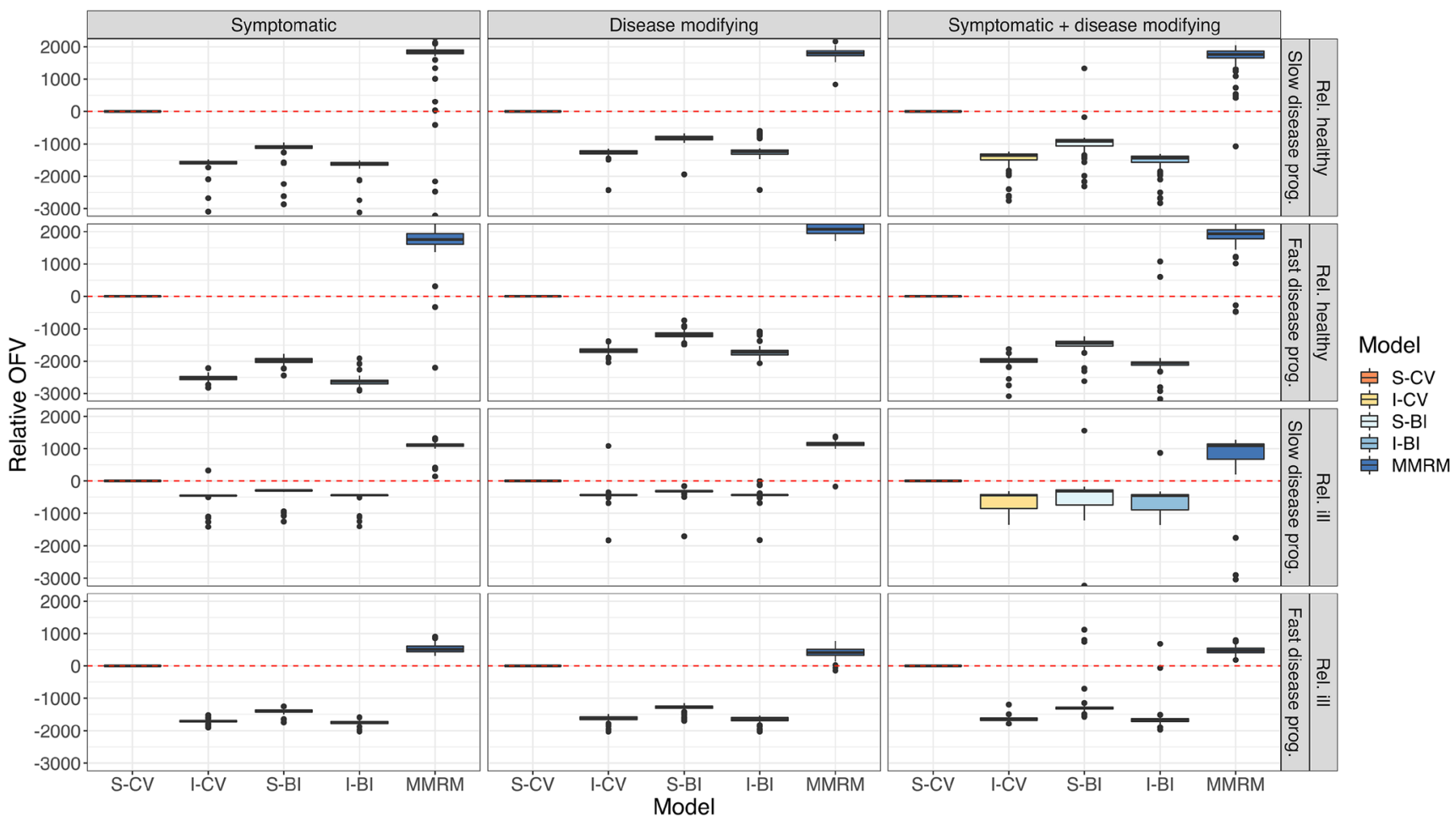

Fig. 6. Relative OFV of the investigated models on the validation data set, stratified by drug effect and population. The standard continuous variable (S-CV) model is used as the reference model. Note that the y-axis has been cut for visibility. IRT, item response theory; I-BI, IRTinformed bounded integer model; I-CV, IRT-informed continuous variable model; S-BI, standard bounded integer model; MMRM, mixed model for repeated measures 

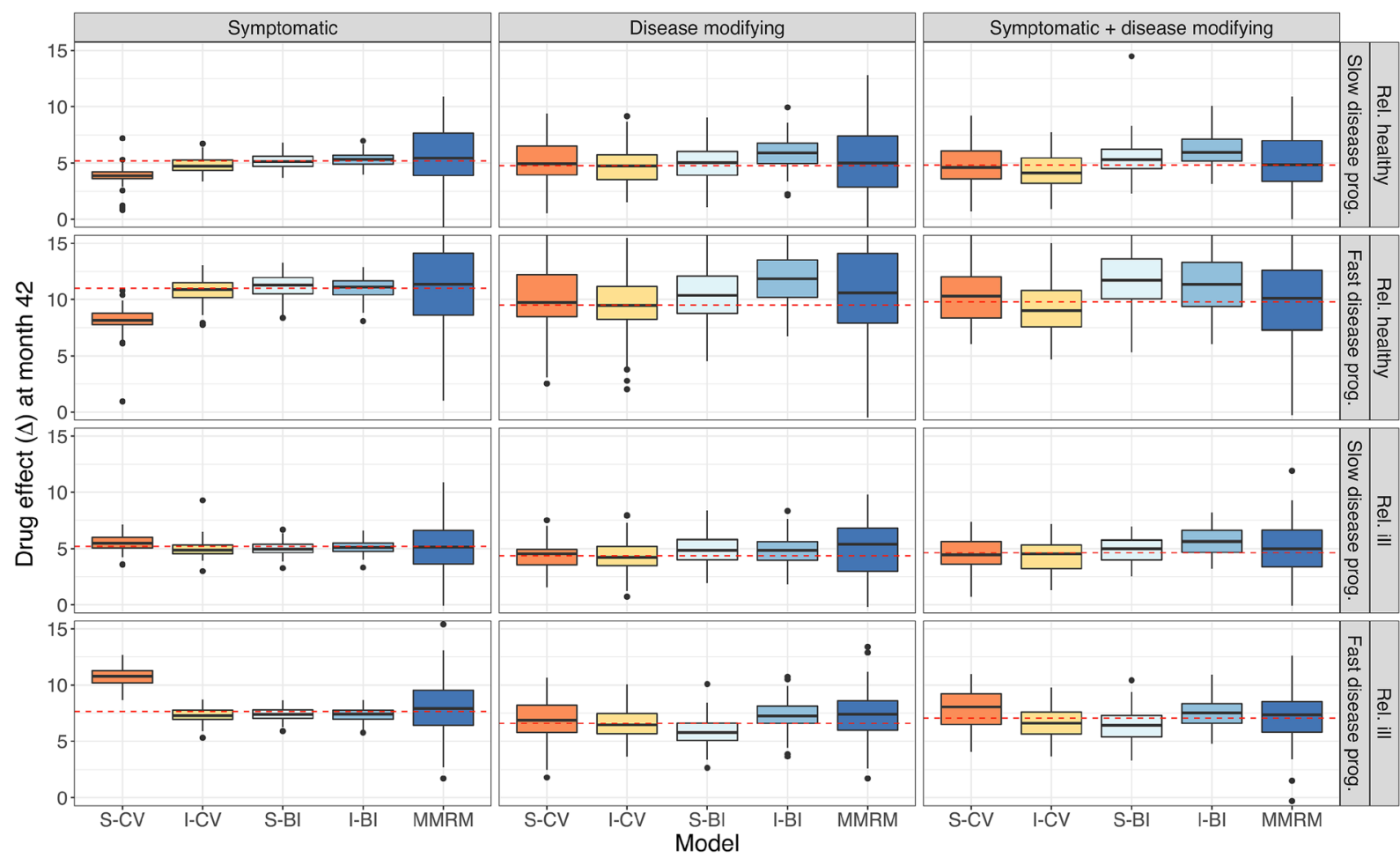

Model

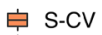

官 I-CV

官

S-BI

官 I-BI

Fig. 7. Predicted and true difference between drug and placebo at end-of-study, stratified by drug effect and population. The boxes represent the distribution of differences across 100 predictions. The dashed red line indicates the true, simulated, average difference in 100 simulations. Note that the $\mathrm{y}$-axis has been cut for visibility. IRT, item response theory; I-BI, IRT-informed bounded integer model; I-CV, IRT-informed continuous variable model; S-BI, standard bounded integer model; S-CV, standard continuous variable model; MMRM, mixed model for repeated measures

the end-of-study, an MMRM model might be the right choice as it is unbiased and structurally simple. However, if anything is to be said about the mechanistic properties of a system or if the model will be used for clinical trial simulations, a full longitudinal model (like CV or BI) should be chosen. The standard BI model is an improvement over the standard CV model, as it respects the bounded data nature and it also allows the simulation of real life-like data. When adding IRTinformed link functions, there was not as clear a difference between I-CV and I-BI, but the simulation properties are still the same. Also, it is then possible to combine information from different model types. This could be valuable when designing new clinical trials. However, the IRT-informed models obviously require that there already exists an IRT model for the scale, which may not always be the case. It should also be a well-constructed IRT model; if it is misspecified, then so will the IRT-informed models be too since the link functions are exact. The pros and cons of the models investigated in this work are summarised in Table I.

Coarsened grid or beta regression models could have been investigated $(15,27)$; coarsened grid models would likely have similar properties to the BI model as they also map the TS to a latent variable. Ordered categorical models could also be applied to composite scale data, but they are parameter heavy and cannot predict data categories not present in the data. They do however respect the boundaries of TS data. In scales with fewer categories one could entertain ordered categorical models, but they are typically not considered for
Table I. The Pros and Cons of Different Methods of Total Score Analysis

\begin{tabular}{lll}
\hline Model & Pros & Cons \\
\hline S-CV & Easy to implement & Does not respect scale
\end{tabular}
boundaries nor data nature, assumes homoscedastic SD

I-CV Better fit than S-CV, allows Requires existing IRT to estimate IRT parameters, model, does not respect unbiased predictions data nature

S-BI Respects data nature and Assumes homoscedastic SD scale boundaries, can (on Z scale) simulate real life-like data

I-BI Better fit than S-BI, respects Requires existing IRT data nature and scale model

boundaries, allows to estimate IRT parameters, can simulate real life-like data, better IRT parameter precision than I-CV

MMRM Unbiased, few mechanistic Many parameters to assumptions needed, robust estimate, does not respect to model misspecification scale boundaries nor data nature, cannot extrapolate

$B I$, bounded integer; $C V$, continuous variable; $I R T$, item response theory; I-BI, IRT-informed BI; I-CV, IRT-informed CV; $M M R M$, mixed models for repeated measures; $Z$, latent variable in BI model 
outcomes with more than maximally 10-20 categories. The properties of a categorical MMRM model, which has been described previously (28) but is not routinely used, would also be highly interesting to explore.

The MMRM model provides a robust alternative where no structural parameters except the mean at each time point and the autoregressive parameters need to be estimated. This means that they are robust to model misspecification. MMRM models are inherently unbiased under dropout if the correct (or an unconstrained) correlation structure is used $(2,29,30)$ and were also unbiased in this work-but had the least precise predictions. Also, the fit was not as good as with the other models in this comparison. This is likely because the AR1 structure could be seen as a model misspecification, as it does not offer the same flexibility as the nonlinear models with IIV, especially IIV on SD. Also, since no slope or offset parameter is estimated, it is not possible to directly make inferences about drug effects in terms of these entities. Model averaging across the investigated models, which is a possible extension of this work, should have revealed the misspecification of MMRM models by rendering low weights. The MMRM models offer large flexibility with respect to the time profile. Unconstrained correlation matrices are conventional, while we used an AR1 matrix. However, both AR1 and unconstrained matrix models were evaluated in $\mathrm{R}$ and provided a similar fit (results not shown). Notably, the AR1 model was much faster to run in NONMEM. We also implemented an additive random effect in the model, which does not constrain the predictions to be positive. We believe this is the standard implementation that MMRM users would adopt in statistical software since a lognormal distribution as used in the S-CV model might not be easy to implement in most software; however, the exponential model was also evaluated in NONMEM (results not shown) with similar results as the additive random effect.

As residuals are one important way to diagnose model misspecification, the expected properties of residuals should be known. For CV models, these have been studied extensively while the discrete data models, like BI, are not as well documented. In this work, we saw that the PIWRES most often gave less than $5 \%$ outliers, defined as $> \pm 2$ SD. This may indicate that the behaviour of the PIWRES metric does not follow that of the standard normal distribution, where $\sim 95 \%$ of the data should be within \pm 2 SD and that perhaps \pm 1.5 SD is more reasonable to use with PIWRES, in order to identify outliers or model misspecification. Alternatively, simulation-based residuals such as normalised prediction distribution errors (NPDE) (31) or quantile residuals (32-34) could be considered, which rescale the residuals to a normal (NPDE) or uniform (quantile) distribution.

The dropout model was only affected by time, and not by disease severity, thus following a missing completely at random (MCAR) mechanism. It is possible that patients who experience severe symptoms of parkinsonism would be more likely to drop out; however, no large differences in dropout rates between treatment and placebo groups were observed in the studies that formed the basis for the overall dropout target of $15 \%$ (19). The MMRM models are valid under missingness at random (MAR). If missingness was not at random (MNAR), a model for the missingness would need to be implemented in the analysis models to avoid bias and imprecision, which can be done in the more mechanistic models, but not in MMRM. This was however out of the scope of this analysis. The amount of dropout also affects the analysis: more dropouts would mean fewer individuals at the end and hence more imprecise estimates for the MMRM model, while the fully longitudinal models handle this phenomenon better, at the cost of higher shrinkage.

We assumed a direct offset effect that was effective immediately after baseline, a simple model, to clearly illustrate the differences between the analysis models. Since the analysis was based (and titrated to the same power via a $t$ test) on the outcome at 42 months, this should have no impact on the results. Also, the number of simulations could have been greater than the 100 used here; however, it was sufficient for detecting trends in the differences in precision and bias between the investigated models.

\section{CONCLUSIONS}

There are many ways to model TS data, and their respective strengths and weaknesses have been highlighted in this work, along with recommendations for when to choose a certain method. For unbiased statistical tests, MMRM appears well suited, but the other methods were more precise in their predictions. For simulations, NLME models, rather than MMRM, need to be chosen. Standard CV models are easy to implement, but BI models are the only ones that respect the discrete data nature. The IRT-informed models (I$\mathrm{CV}$ and I-BI) models provided the best fit and also the best performance on external data. Furthermore, the IRT link functions allow IRT parameters to be retrieved with high precision and low bias-especially in the I-BI model. This will aid modellers analysing clinical trial data with total scores to choose a fit-for purpose analysis method.

\section{SUPPLEMENTARY INFORMATION}

The online version contains supplementary material available at https://doi.org/10.1208/s12248-020-00546-w.

\section{FUNDING}

Open access funding provided by Uppsala University. This work was financially supported by the Swedish Research Council Grant 2018-03317.

\section{SUPPLEMENTARY INFORMATION}

The online version contains supplementary material available at https://doi.org/10.1208/s12248-020-00546-w.

Open Access This article is licensed under a Creative Commons Attribution 4.0 International License, which permits use, sharing, adaptation, distribution and reproduction in any medium or format, as long as you give appropriate credit to the original author(s) and the source, provide a link to the Creative Commons licence, and indicate if changes were made. The images or other third party material in this article 
are included in the article's Creative Commons licence, unless indicated otherwise in a credit line to the material. If material is not included in the article's Creative Commons licence and your intended use is not permitted by statutory regulation or exceeds the permitted use, you will need to obtain permission directly from the copyright holder. To view a copy of this licence, visit http://creativecommons.org/licenses/by/4.0/.

\section{REFERENCES}

1. Wellhagen GJ, Kjellsson MC, Karlsson MO. A bounded integer model for rating and composite scale data. AAPS J. 2019;21(4):74.

2. Mallinckrodt $\mathrm{CH}$, Clark WS, David SR. Accounting for dropout bias using mixed-effects models. J Biopharm Stat. 2001;11(12):9-21.

3. Mallinckrodt CH, Lane PW, Schnell D, Peng Y, Mancuso JP. Recommendations for the primary analysis of continuous endpoints in longitudinal clinical trials. Drug Inf J. 2008;42(4):303-19.

4. Siddiqui O, Hung HMJ, O'Neill R. MMRM vs. LOCF: a comprehensive comparison based on simulation study and 25 NDA datasets. J Biopharm Stat. 2009;19(2):227-46.

5. Smithson M, Verkuilen J. A better lemon squeezer? Maximumlikelihood regression with beta-distributed dependent variables. Psychol Methods. 2006;11(1):54-71.

6. Ahamadi M, Conrado DJ, Macha S, Sinha V, Stone J, Burton J, et al. Development of a disease progression model for Leucinerich repeat kinase 2 in Parkinson's disease to inform clinical trial designs. Clin Pharmacol Ther. 2020;107(3):553-62.

7. Liu D, Zhang Y, Jiang J, Choi J, Li X, Zhu D, et al. Translational modeling and simulation in supporting earlyphase clinical development of new drug: a learn-researchconfirm process. Clin Pharmacokinet. 2017;56(8):925-39.

8. Ospina R, Ferrari SLP. Inflated beta distributions. Stat Pap. 2008;51(1):111.

9. Ospina R, Ferrari SLP. A general class of zero-or-one inflated beta regression models. Comput Stat Data Anal. 2012;56(6):1609-23.

10. Galvis DM, Bandyopadhyay D, Lachos VH. Augmented mixed beta regression models for periodontal proportion data. Stat Med. 2014;33(21):3759-71.

11. Figueroa-Zúñiga JI, Arellano-Valle RB, Ferrari SLP. Mixed beta regression: a Bayesian perspective. Comput Stat Data Anal. 2013;61:137-47.

12. Brisco AMD, Migliorati S. A new mixed-effects mixture model for constrained longitudinal data. Stat Med. 2020;39(2):129-45.

13. Hu C, Adedokun OJ, Zhang L, Sharma A, Zhou H. Modeling near-continuous clinical endpoint as categorical: application to longitudinal exposure-response modeling of Mayo scores for golimumab in patients with ulcerative colitis. J Pharmacokinet Pharmacodyn. 2018;45(6):803-16.

14. $\mathrm{Hu} \mathrm{C}$, Randazzo B, Sharma A, Zhou H. Improvement in latent variable indirect response modeling of multiple categorical clinical endpoints: application to modeling of guselkumab treatment effects in psoriatic patients. J Pharmacokinet Pharmacodyn. 2017;44(5):437-48.

15. Lesaffre E, Rizopoulos D, Tsonaka R. The logistic transform for bounded outcome scores. Biostat Oxf Engl. 2007;8(1):72-85.

16. Donohue MC, Aisen PS. Mixed model of repeated measures versus slope models in Alzheimer's disease clinical trials. J Nutr Health Aging. 2012;16(4):360-4.
17. Chen Y-F, Ni X, Fleisher AS, Zhou W, Aisen P, Mohs R. A simulation study comparing slope model with mixed-model repeated measure to assess cognitive data in clinical trials of Alzheimer's disease. Alzheimers Dement N Y N. 2018;4:46-53.

18. Buatois S, Retout S, Frey N, Ueckert S. Item response theory as an efficient tool to describe a heterogeneous clinical rating scale in de novo idiopathic Parkinson's disease patients. Pharm Res. 2017;34(10):2109-18.

19. Zagmutt FJ, Tarrants ML. Indirect comparisons of adverse events and dropout rates in early Parkinson's disease trials of pramipexole, ropinirole, and rasagiline. Int $\mathrm{J}$ Neurosci. 2012;122(7):345-53.

20. Hooker AC, Staatz CE, Karlsson MO. Conditional weighted residuals (CWRES): a model diagnostic for the FOCE method. Pharm Res. 2007;24(12):2187-97.

21. Agresti A. Logit models for multinomial responses. In: Categorical data analysis. 2nd ed. Hoboken, NJ: John Wiley \& Sons; 2002.

22. Lindbom L, Ribbing J, Jonsson EN. Perl-speaks-NONMEM (PsN)-a Perl module for NONMEM related programming. Comput Methods Prog Biomed. 2004;75(2):85-94.

23. Lindbom L, Pihlgren P, Jonsson EN, Jonsson N. PsN-toolkit-a collection of computer intensive statistical methods for nonlinear mixed effect modeling using NONMEM. Comput Methods Prog Biomed. 2005;79(3):241-57.

24. R Core Team. R: a language and environment for statistical computing. Vienna, Austria: R Foundation for Statistical Computing; 2019. Available from: https://www.R-project.org/

25. Wickham H, Averick M, Bryan J, Chang W, McGowan L, François R, et al. Welcome to the Tidyverse. J Open Source Softw. 2019:4(43):1686.

26. UUPharmacometrics/piraid. Uppsala University, Pharmacometrics Research Group; 2020 [cited 2020 Apr 14]. Available from: https://github.com/UUPharmacometrics/piraid

27. Conrado DJ, Denney WS, Chen D, Ito K. An updated Alzheimer's disease progression model: incorporating nonlinearity, beta regression, and a third-level random effect in NONMEM. J Pharmacokinet Pharmacodyn. 2014;41(6):581-98.

28. Jansen I, Beunckens C, Molenberghs G, Verbeke G, Mallinckrodt C. Analyzing incomplete discrete longitudinal clinical trial data. Stat Sci. 2006;21(1):52-69.

29. Mallinckrodt CH, Sanger TM, Dubé S, DeBrota DJ, Molenberghs G, Carroll RJ, et al. Assessing and interpreting treatment effects in longitudinal clinical trials with missing data. Biol Psychiatry. 2003;53(8):754-60.

30. Mallinckrodt CH, Kaiser CJ, Watkin JG, Molenberghs G, Carroll RJ. The effect of correlation structure on treatment contrasts estimated from incomplete clinical trial data with likelihood-based repeated measures compared with last observation carried forward ANOVA: Clin Trials. 2016.

31. Comets E, Brendel K, Mentré F. Computing normalised prediction distribution errors to evaluate nonlinear mixedeffect models: the npde add-on package for R. Comput Methods Prog Biomed. 2008;90(2):154-66.

32. Dunn PK, Smyth GK. Randomized Quantile residuals. J Comput Graph Stat. 1996;5(3):236-44.

33. Gelman A, Hill J. Data analysis using regression and multilevel/ hierarchical models: Cambridge University Press; 2006.

34. Hartig F. DHARMa: Residual diagnostics for hierarchical (multi-level/mixed) regression models. 2020. Available from: http://florianhartig.github.io/DHARMa/

Publisher's Note Springer Nature remains neutral with regard to jurisdictional claims in published maps and institutional affiliations. 